\title{
Corrigendum: An MRTF-A-Sp1-PDE5 Axis Mediates Angiotensin-II-Induced Cardiomyocyte Hypertrophy
}

\author{
Teng Wu ${ }^{1}$, Huidi Wang ${ }^{1}$, Xiaojun Xin ${ }^{3}$, Tianyi Zhang ${ }^{1}$, Jie Yang ${ }^{1}$, Yannan Hou ${ }^{1}$, \\ Mingming Fang ${ }^{2,4 *}$, Xiang $\mathrm{Lu}^{3 *}$ and Yong $\mathrm{X} \mathrm{U}^{1,4 *}$
}

OPEN ACCESS

Approved by:

Frontiers Editorial Office,

Frontiers Media SA, Switzerland

${ }^{*}$ Correspondence:

Mingming Fang

dafeifang@163.com

Xiang Lu

luxiang66@njmu.edu.cn

Yong Xu

yjxu@njmu.edu.cn

Specialty section:

This article was submitted to

Molecular Medicine

a section of the journa

Frontiers in Cell and Developmental

Biology

Received: 19 May 2021

Accepted: 26 May 2021

Published: 29 June 2021

Citation:

Wu T, Wang $H$, Xin $X$, Zhang T, Yang J, Hou Y, Fang M, Lu X and Xu Y (2021)

Corrigendum: An MRTF-A-Sp1-PDE5

Axis Mediates Angiotensin-II-Induced

Cardiomyocyte Hypertrophy.

Front. Cell Dev. Biol. 9:711764.

doi: 10.3389/fcell.2021.711764
${ }^{1}$ Key Laboratory of Targeted Intervention of Cardiovascular Disease and Collaborative Innovation Center for Cardiovascular Translational Medicine, Department of Pathophysiology, Nanjing Medical University, Nanjing, China, ${ }^{2}$ Laboratory Center for Experimental Medicine, Department of Clinical Medicine, Jiangsu Health Vocational College, Nanjing, China, ${ }^{3}$ Department of Geriatrics, Sir Run Run Hospital, Nanjing Medical University, Nanjing, China, ${ }^{4}$ Institute of Biomedical Research, Liaocheng University, Liaocheng, China

Keywords: cardiac hypertrophy, cardiomyocyte, transcriptional regulation, angiotensin II, MRTF-A, Sp1, PDE5

\section{A Corrigendum on}

An MRTF-A-Sp1-PDE5 Axis Mediates Angiotensin-II-Induced Cardiomyocyte Hypertrophy by Wu, T., Wang, H., Xin, X., Zhang, T., Yang, J., Hou, Y., et al. (2020). Front. Cell Dev. Biol. 8:839. doi: $10.3389 /$ fcell.2020.00839

Tianyi Zhang was not included as an author in the published article. The corrected Author Contributions Statement appears below.

"YX and TW conceived the project, designed the experiments, and wrote the manuscript. TW, HW, XX, TZ, JY, and YH performed experiments and collected data. MF and XL secured funding and provided supervision. All authors contributed to the article and approved the submitted version."

The authors apologize for this error and state that this does not change the scientific conclusions of the article in any way. The original article has been updated.

Copyright $\odot 2021 \mathrm{Wu}$, Wang, Xin, Zhang, Yang, Hou, Fang, Lu and Xu. This is an open-access article distributed under the terms of the Creative Commons Attribution License (CC BY). The use, distribution or reproduction in other forums is permitted, provided the original author(s) and the copyright owner(s) are credited and that the original publication in this journal is cited, in accordance with accepted academic practice. No use, distribution or reproduction is permitted which does not comply with these terms. 